\title{
Randomized Prospective Evaluation of Nephrostomy Tube Configuration: Impact on Postoperative Pain
}

\author{
Derek Weiland, Renato N. Pedro, J. Kyle Anderson, Sara L. Best, Courtney Lee, Kari Hendlin, \\ Johnstone Kim, Manoj Monga
}

Department of Urologic Surgery, University of Minnesota, Minneapolis, USA

\begin{abstract}
Objective: Conduct a prospective randomized single-blind comparison of two nephrostomy catheter designs, evaluating specifically intraoperative placement and postoperative comfort.

Materials and Methods: The single-blind, prospective randomized trial was conducted with institutional review board approval. All patients undergoing percutaneous nephrolithotomy who gave informed consent were randomized to placement of either a Boston Scientific Flexima 8.3F pigtail nephrostomy tube \#27-180 (PIG) or a Boston Scientific 8.2F nephroureteral stent \#410-126 (NUS). Randomization was concealed from the surgeon until time of placement. Subjective intraoperative placement characteristics were rated by the surgeon on a scale of $1=$ excellent, $2=$ fair, $3=$ good and $4=$ poor. The patient's postoperative pain intensity was evaluated with a Visual Analog Pain Score $(0=$ no pain to $10=$ worst pain $)$.

Results: Nine patients were randomized to each group. The PIG group was rated significantly better than the NUS group with regards to ease of placement $(\mathrm{p}=0.007)$ and radiopacity of the tube $(\mathrm{p}=0.007)$ by surgeon. Visual analog pain scores on postoperative day one, was significantly lower in the PIG group $($ mean $=2+/-2)$ than the NUS group $($ mean $=5+/-1)(p=$ 0.004). The mean amount of intra-venous morphine equivalent given in the PIG group (mean $=1+/ 4$ Eq morphine) was less on average compared to the NUS group (mean $=6+/ 13 \mathrm{Eq}$ morphine), but the differences did not reach statistical significance $(\mathrm{p}=0.06)$.

Conclusions: Following percutaneous nephrolithotomy, use of a small pig-tail nephrostomy tube results in greater ease of placement and less postoperative pain than a nephroureteral catheter.
\end{abstract}

Key words: percutaneous nephrolithotomy; postoperative pain; nephrostomy; stent; randomized controlled trial Int Braz J Urol. 2007; 33: 313-22

\section{INTRODUCTION}

Percutaneous nephrolithotomy (PCNL) has been the standard of care for large renal calculi since its introduction in 1976 (1). It has been reported that patients will require up to 6.5 doses of narcotics postoperative, and the time to complete recuperation and resumption of full activities is over 9 days $(2,3)$.
It has been proposed that the degree of postoperative discomfort corresponds to the size of percutaneous nephrostomy tube left at the conclusion of the procedure (4). However, while catheter size may impact patient discomfort, the impact of catheter configuration on postoperative pain has not been evaluated in a prospective study. 
The purpose of this study is to compare patient discomfort, morbidity and efficacy with two $8 \mathrm{~F}$ catheters: a pigtail (Flexima) versus an open-ended nephroureteral catheter for postoperative renal drainage following PCNL. This study will specifically evaluate the impact of nephrostomy tube design.

\section{MATERIALS AND METHODS}

This single-blind, prospective randomized trial was conducted with institutional review board approval. All patients undergoing percutaneous nephrolithotomy from January 2005 to March 2006 who gave informed consent were randomized to placement of either a Boston Scientific Flexima 8.3F pigtail nephrostomy tube (Flexima copolymers, \#27-180) (PIG) or a Boston Scientific 8.2F nephroureteral stent (Percuflex durometer, \#410-126) (NUS). Patients were blinded to the stent placed for the duration of the study. Simple randomization was performed using a computergenerated number sequence and was concealed from the surgeon until time of placement.

All patients undergoing percutaneous nephrolithotomy during the study period were approached for informed consent to participate. Patients were excluded if an antegrade endopyelotomy was performed concurrently. Patients were counseled that if a ureteral injury occurred during the procedure a nephroureteral stent would be left in place, however this did not occur during our study.

Placement of each catheter was performed over the working super-stiff guide-wire, through the lumen of the $30 \mathrm{~F}$ Amplatz nephrostomy sheath at the completion of the procedure. The position of the catheter was confirmed fluoroscopically with the pigtail nephrostomy catheter positioned in the renal pelvis, while the nephroureteral stent traversed the ureter with coiling in the bladder. The coils for each catheter were formed by gentle tension on the retention suture. The catheters were secured at the skin level with 2-0 silk sutures.

Subjective intraoperative placement characteristics were rated by the surgeon on a scale of $1=$ excellent, $2=$ fair, $3=\operatorname{good}$ and $4=$ poor. The patient's postoperative pain intensity was evaluated with a Visual Analog Pain Score $(0=$ no pain to $10=$ worst pain). Narcotic diaries were maintained and all opioid drugs were converted to a morphine equivalent (Eq morphine), Table-1.

The present study was powered based on prior studies of percutaneous nephrostomy size and technique, which have demonstrated significant differences with sample sizes of 10 patients per arm (4-6).

Student t-test was also used to study the impact of catheter configuration on pulmonary function (FEV1), blood loss (Hgb), serum creatinine level (Crt), length of stay, and time to ambulation and oral food intake (hours). It is relevant to mention that oral fluid intake was encouraged to commence in the immediate peri-operative recovery period, and time to oral intake was defined as tolerating oral liquids. $P$ values $<0.05$ were considered significant.

Nephrostomy catheters were clamped on postoperative day 1 if there were no concerns regarding fever, pain, hematuria or residual stone fragments. If the patient did not experience pain within 3 hours of clamping, the catheter was removed by transecting the catheter at the skin level to release the retention coil.

\section{RESULTS}

Nine patients were randomized to each group. Demographic features of both groups (gender, stone size, laterality) were comparable except mean age, which was significantly lower in the NUS than PIG $(54 \pm 11$ versus $65 \pm 11$ years, respectively) $(p=0.004)$ (Table-2).

Table 1 - Calculating morphine equivalent dose: Total EDU $\times$ morphine $30 \mathrm{mg}=$ morphine equivalent dose.

Opioid

Equianalgesic Dose ED (mg)

\begin{tabular}{lr} 
Oxycodone & 20 \\
Hydrocodone & 30 \\
Codeine & 200 \\
Meperidine & 300 \\
\hline
\end{tabular}


Table 2 - Demographic features of the $8.3 F$ pigtail nephrostomy tube (PIG) and the 8.2F nephroureteral stent (NUS) groups.

\begin{tabular}{lccc}
\hline & PIG (range) & NUS (range) & p Value \\
\hline Age (years) & $65 \pm 11(51-78)$ & $54 \pm 11(36-69)$ & $0.04^{*}$ \\
Male / Female & $6 / 2$ & $6 / 5$ & 0.34 \\
Stone size $\left(\mathrm{cm}^{2}\right)$ & $6.7 \pm 1.2(2.5-7.5)$ & $3.2 \pm 1.3(1.6-6.0)$ & 0.15 \\
Left / Right Side & $3 / 5$ & $8 / 3$ & 0.14 \\
BMI $\left(\mathrm{kg} / \mathrm{m}^{2}\right)$ & $30 \pm 7(21.2-42.9)$ & $29 \pm 6(19.1-40.1)$ & 0.40 \\
Previous PCNL & 4 & 4 & 0.58 \\
\hline
\end{tabular}

The PIG group was rated significantly better than the NUS group with regards to ease of placement $(\mathrm{p}=0.007)$ and radiopacity of the tube $(\mathrm{p}=0.007)$ by surgeon (Figure-1).

Visual analog pain scores on postoperative day one, was significantly lower in the PIG group (mean $=2 \pm 2)$ than the NUS group (mean $=5 \pm 1)(\mathrm{p}=$ 0.004 ) (Figure-2). The mean amount of intra-venous morphine equivalent given in the PIG group $($ mean $=$ 1, Standard Deviation $=4$ Eq morphine) was less on average compared to the NUS group (mean $=6$, Standard Deviation $=13 \mathrm{Eq}$ morphine), but the differences did not reach statistical significance $(\mathrm{p}=$ $0.06)$. In the PIG group five of eight patients $(62.5 \%)$ had their catheters placed supra-costal versus only 3 of eleven $(27.7 \%)$ in the NUS group. There was no significant difference in calyx selected for puncture in the PIG ( 2 upper, 4 middle and 2 lower) and NUS (3 upper, 2 middle, 6 lower) groups. The location of puncture did not influence the ease of placement in both groups.

One patient of NUS group was excluded from the time to ambulation and PO intake statistical analysis because of a pulmonary embolus that required rest and fasting for a longer period of time (120 hours).

PIG group had significant less time $(\mathrm{p}=0.005)$ to PO intake than NUS group $(3.7 \pm 2.8$ hours PIG -

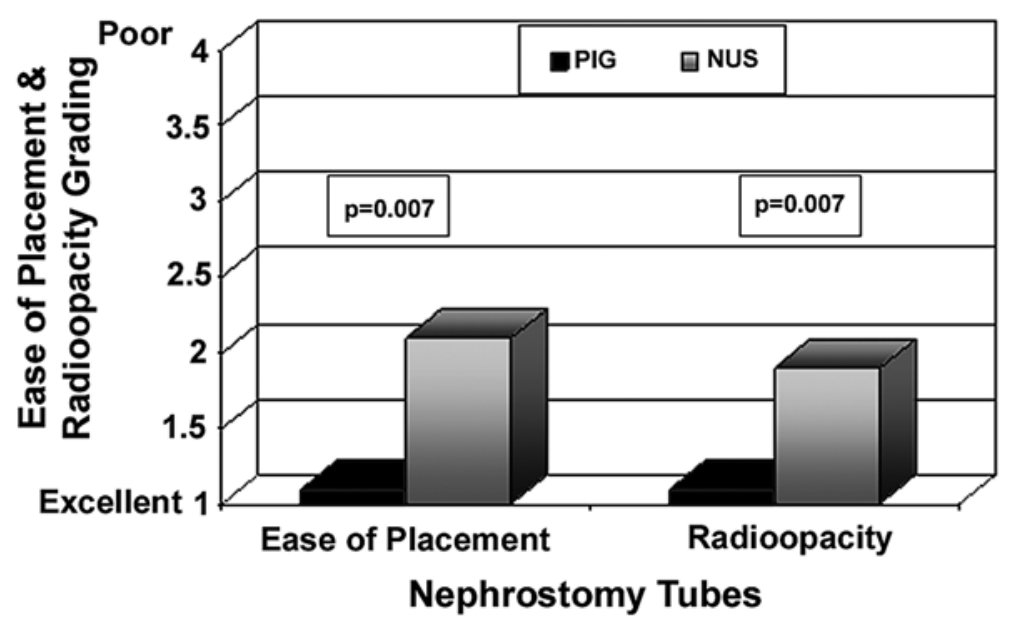

Figure 1 - Surgeon's rating of nephrostomy tube ease of placement and radiopacity. 


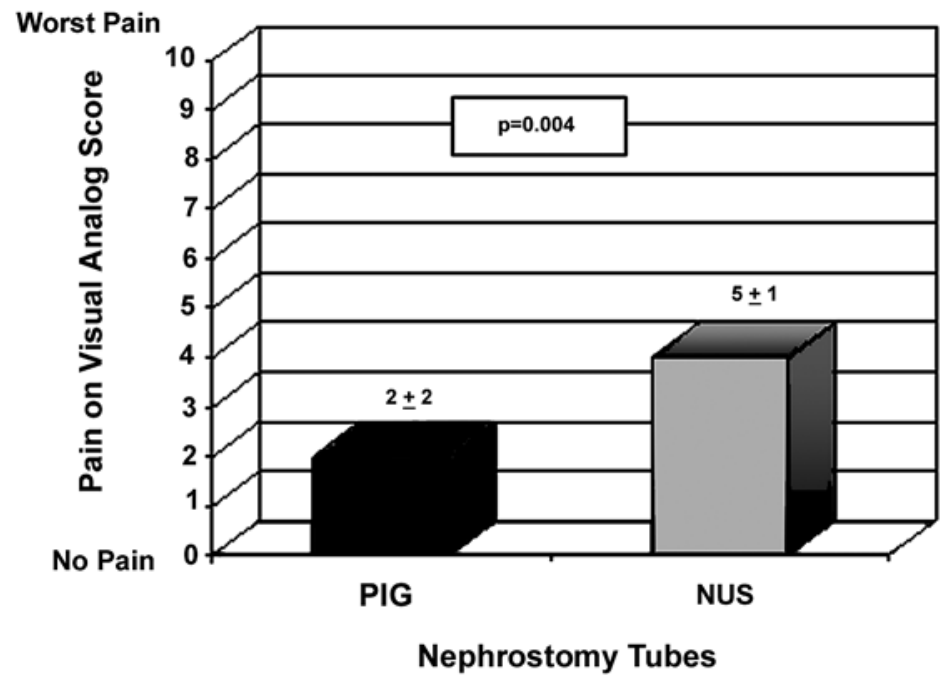

Figure 2 - Postoperative day-1 pain.

$10.88 \pm 6.19$ hours NUS). Neither catheter was superior in regards to change in $\mathrm{Hgb}(0.96 \pm 0.53 \mathrm{mg} /$ dL NUS, $2.16 \pm 0.53 \mathrm{mg} / \mathrm{dL}$ PIG) $(\mathrm{p}=0.15)$; change in Crt $(0.047 \pm 0.57 \mathrm{mg} / \mathrm{dL}$ NUS, $0.14 \pm 0.22 \mathrm{mg} / \mathrm{dL}$ PIG) $(\mathrm{p}=0.20)$; change in FEV1 $(88.9 \pm 32.02 \mathrm{~mL}$ NUS $-243.6 \pm 119.89 \mathrm{~mL}$ PIG) $(\mathrm{p}=0.60)$; length of stay $(2.81 \pm 1.82$ days NUS $-3.15 \pm 3.44$ days PIG) $(\mathrm{p}=0.80)$; dressing changes $(6.8 \pm 5.90 \mathrm{NUS}-3.75 \pm$
$2.90 \mathrm{PIG})(\mathrm{p}=0.38)$, or ambulation $(17.50 \pm 10.53$ hours NUS $-9.7 \pm 6.0$ hours PIG) $(\mathrm{p}=0.11)($ Table-3).

\section{COMMENTS}

Percutaneous nephrostomy tubes are associated with postoperative pain and long hospital

Table 3 - Pre and postoperative data for the $8.3 F$ pigtail nephrostomy tube $(P I G)$ and the $8.2 F$ nephroureteral stent (NUS) groups.

\begin{tabular}{|c|c|c|c|c|c|c|c|}
\hline \multirow[b]{2}{*}{ Average } & \multicolumn{2}{|c|}{ PIG (range) } & \multicolumn{2}{|c|}{ NUS (range) } & \multicolumn{2}{|c|}{ Postop Change } & \multirow{2}{*}{$\frac{\text { pValue }}{-}$} \\
\hline & Preop & Postop & Preop & Postop & NUS & PIG & \\
\hline $\operatorname{Hgb}(\mathrm{mg} / \mathrm{dL})$ & $\begin{array}{c}12.97 \\
(10.1-14.8)\end{array}$ & $\begin{array}{c}10.35 \\
(8.9-14.8)\end{array}$ & $\begin{array}{c}12.36 \\
(10.4-14.7)\end{array}$ & $\begin{array}{l}11.41 \\
(8-15)\end{array}$ & 0.96 & 2.16 & 0.15 \\
\hline $\operatorname{Crt}(\mathrm{mg} / \mathrm{dL})$ & $\begin{array}{c}1.09 \\
(0.7-1.4)\end{array}$ & $\begin{array}{c}1.24 \\
(1.0-1.6)\end{array}$ & $\begin{array}{c}1.19 \\
(0.6-2.6)\end{array}$ & $\begin{array}{c}1.14 \\
(0.7-2.6)\end{array}$ & 0.05 & 0.14 & 0.20 \\
\hline FEV1 (mL) & $\begin{array}{c}1978.0 \\
(700-3500)\end{array}$ & $\begin{array}{c}1734.3 \\
(700-3500)\end{array}$ & $\begin{array}{c}1445.0 \\
(800-2000)\end{array}$ & $\begin{array}{c}1361.1 \\
(800-2000)\end{array}$ & 88.9 & 243.6 & 0.60 \\
\hline Ambulation (h) & 9.7 & 21) & 17.5 & -120) & & - & 0.11 \\
\hline Hospital stay (d) & 3.15 & 10) & 2.81 & $(-7)$ & & 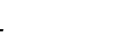 & 0.80 \\
\hline PO intake (h) & 3.7 & $-8)$ & 6.19 & $-18)$ & & 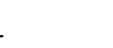 & $0.05^{*}$ \\
\hline Dressings (n) & 3.75 & $-8)$ & 6.80 & $-15)$ & & - & 0.38 \\
\hline
\end{tabular}

$H g b=$ hemoglobin Crt $=$ creatinine $; F E V=$ forced expiratory volume . 
stays. The intensity of distress is related to the size of the nephrostomy catheters. It has been reported that smaller catheters reduce patient discomfort without increasing procedure morbidity (5).

The issue of stent size (8F vs. 20F or larger) has already been addressed clearly in the literature $(5,6)$ and currently our practice is to utilize exclusively an $8 \mathrm{~F}$ drainage tube after PCNL. Tubeless PCNL has been shown to be an effective alternative in select patients (6), though one might debate the relative quality of life issues related to a PNT vs. ureteral stent left following a tubeless procedure. Recently, Choi et al. demonstrated in a randomized trial that the post operative discomfort is similar between small bore nephrostomy tubes and tubeless PCNL (7). Though convalescence was shorter for the tubeless cohort in this study, the nephrostomy tube was left in place for 3-5 days, which exceeds our practice of removing the tube within the first 48 hours if possible.

In our minds, the remaining issue to resolve was to determine the optimal configuration of the percutaneous nephrostomy catheter. Though one might intuitively predict a pigtail catheter would cause less discomfort, lacking the ureteral and bladder segments, an alternative view would be that the nephroureteral stent promotes drainage, particularly in the face of clot or residual stone fragments. Nephrostomy catheter configuration has previously been demonstrated to impact drainage properties and retention strength (8).

Whereas scientific reports have analyzed specifically the size of the PNT and its relationship to postoperative pain, no study has prospectively compared different types of mini-tubes of the same size with regards to patient discomfort.

In this study, 18 patients were randomized and prospectively analyzed regarding severity of pain and opioid intake after placement of two different 8.3F. Catheters; a pigtail (PIG) and nephroureteral (NUS) for nephrostomy drainage. Visual analog pain scores demonstrated that the PIG group had significantly less postoperative pain than the NUS group $(\mathrm{p}=0.04)$. The same trend was noted with regards to intra-venous morphine equivalent required by PIG group, which was less on average than NUS ( $p=0.06)$. Surprisingly, PIG group had a higher incidence of supra-costal punctures, which would predispose to more pain, therefore the differences between NUS and PIG may be even more pronounced than noted in our results.

None of the groups showed significant differences in blood loss, length of hospital stay, pulmonary function and time to ambulation; these data are supported by recent reports that present small bore catheters as a safe and comfortable choice for nephrostomy drainage $(5,6)$. Time to PO intake was significantly lower in the PIG group than the NUS (p $=0.05$ ), which may be linked to the difference in pain described above.

In addition, both catheters were evaluated by the urologic surgeons according to the ease of placement and radiopacity. Although both catheters demanded no great effort in their insertion, the PIG catheters were found to be easier to manipulate and easier to be visualized under fluoroscopy than NUS $(\mathrm{p}=0.007)$.

In a different patient population, Mokhmalji et al. presented a prospective randomized clinical trial comparing quality of live (QoL) after ureteral (JJ) stent or nephrostomy tube placement for relief of ureteral obstruction. They demonstrated that reduction in $\mathrm{QoL}$ was moderate but more pronounced in patients with ureteral stents than in those who underwent percutaneous nephrostomy (9). This study supports our observation that patient discomfort increases when the drainage catheter traverses the ureter and bladder; for this reason, we currently use a nephroureteral stent only in the face of endopyelotomy or ureteral perforation.

In contrast, Karger et al. stated that the $8.5 \mathrm{~F}$ NUS spared patients of post operative pain compared to a historical control group comprised of patients with a 24F Malecot nephrostomy tube (10). This study, however, was not randomized and used catheters of greatly differing calibers for their comparison; such that the observed differences could be attributable to catheter size rather than configuration.

One limitation of the present study is that we did not assess other urinary tract symptoms such as urgency and frequency, nor did we localize the site of the pain. Nevertheless, as our study evaluates the impact on post operative pain and morbidity of two catheters of the same size, the findings suggest that 
nephrostomy catheter design may play an important role in patient postoperative comfort.

\section{CONCLUSION}

Following percutaneous nephrolithotomy, use of a small pig-tail nephrostomy tube results in greater ease of placement and less postoperative pain than a nephroureteral catheter.

\section{CONFLICT OF INTEREST}

The research was supported by Boston Scientific.

\section{REFERENCES}

1. Fernstrom I, Johansson B: Percutaneous pyelolithotomy. A new extraction technique. Scand J Urol Nephrol. 1976; 10: 257-9.

2. Rittenberg MH, Koolpe H, Keeler L, McNamara T, Bagley DH: Pain control: comparison of percutaneous and operative nephrolithotomy. Urology. 1985; 25: 468-71.

3. Netto NR Jr, Lemos GC, De Almeida Claro JF, Palma PC: Comparison between percutaneous nephrolithotomy and open stone procedures. Int Urol Nephrol. 1988; 20: $225-30$
4. Maheshwari PN, Andankar MG, Bansal M: Nephrostomy tube after percutaneous nephrolithotomy: large-bore or pigtail catheter? J Endourol. 2000; 14: 735-7; discussion 737-8.

5. Pietrow PK, Auge BK, Lallas CD, Santa-Cruz RW, Newman GE, Albala DM, et al.: Pain after percutaneous nephrolithotomy: impact of nephrostomy tube size. J Endourol. 2003; 17: 411-4.

6. Desai MR, Kukreja RA, Desai MM, Mhaskar SS, Wani $\mathrm{KA}$, Patel SH, et al.: A prospective randomized comparison of type of nephrostomy drainage following percutaneous nephrostolithotomy: large bore versus small bore versus tubeless. J Urol. 2004; 172:565-7.

7. Choi M, Brusky J, Weaver J, Amantia M, Bellman GC: Randomized trial comparing modified tubeless percutaneous nephrolithotomy with tailed stent with percutaneous nephrostomy with small-bore tube. J Endourol. 2006; 20: 766-70.

8. Canales BK, Hendlin K, Braasch M, Antolak C, Reddy A, Odeh B, et al.: Percutaneous nephrostomy catheters: drainage flow and retention strength. Urology. 2005; 66:261-5.

9. Mokhmalji H, Braun PM, Martinez Portillo FJ, Siegsmund M, Alken P, Kohrmann KU: Percutaneous nephrostomy versus ureteral stents for diversion of hydronephrosis caused by stones: a prospective, randomized clinical trial. J Urol. 2001; 165: 1088-92.

10. Kader AK, Finelli A, Honey RJ: Nephroureterostomydrained percutaneous nephrolithotomy: modification combining safety with decreased morbidity. J Endourol. 2004; 18: 29-32.

\section{Correspondence address:}

Dr. Manoj Monga

Department of Urologic Surgery

University of Minnesota

Mayo 394, 420 Delaware St. SE

Minneapolis, MN, 55455, USA

Fax: + $1612624-4430$

E-mail: endourol@yahoo.com 


\section{EDITORIAL COMMENT}

The era of open stone surgery and plastic surgical correction of the renal pelvis, beside the unavoidable trauma of open surgical access had already been dominated by postoperative problems regarding drainage systems and urinary reduction.

Postoperative problems due to different percutaneous and/or intraluminal catheters basically are induced by the following factors: affection of the respiratory system caused by transpassing catheters, affection of the percutaneous stent location because of the renal mobility, suppression of the peristaltic activity inside the collecting system caused by the"foreign bodies", decline in reflux - protection caused by ureteral stents entering the bladder and passing Waldeyers sheet.

The effect on patients postoperative condition means additional multiple branch therapy, i.e. analgesic regimen, treatment of ascending infections,

\section{EDITORIAL COMMENT}

One of the most significant areas of investigation with regards to percutaneous nephrolithotomy (PCNL) involves the need for, and type of drainage of the collecting system with a percutaneous nephrostomy catheter. Postoperative discomfort from a percutaneous catheter placed at the conclusion of the procedure has been attributed to a number of factors including catheter diameter, access location, and number of catheters. Many surgeons have been revising their technique in favor of placing smaller catheters or performing tubeless PCNL, with the intention of reducing patient discomfort without compromising procedural outcomes or increasing complications. prolongation of the hospital stay and an increase in costs as well.

The urological progress of the last decade especially with minimally invasive procedures and also minimal percutaneous nephrolithotripsy (PCNL) induced effective research and investigations to minimize the postoperative drainage related trauma, what has also been demonstrated by the present article, culminating in the tubeless postoperative nephrostomy using the pouring effect of gelatine to perform a watertight closure of the working channel.

The very actual status of the investigations demands for obligatory strategies and roules also for drainage systems after PCNL dependant on residual clearance function, stone history (number, size, consistency ), infectious conditions, duration of the surgical procedure and patients complication level value.

Dr. Volker Schick

Urologische Abteilung

Robert Koch-Krankenhaus

Gehrden, Germany

E-mail:schick@rkk-gehrden.de

The purpose of this study was to prospectively examine the impact of percutaneous catheter design on postoperative pain following PCNL. Specifically, an $8.3 \mathrm{~F}$ pigtail nephrostomy catheter (PIG) was compared to an $8.2 \mathrm{~F}$ open-ended nephroureteral catheter (NUS). The authors conclude that the PIG was less painful than the NUS, based on lower visual analog pain scores and lower analgesic requirements. Increased pain in the NUS group was thought to be due to the catheter traversing the ureter and bladder. This idea has been previously proposed in studies comparing the discomfort associated with a percutaneous nephrostomy catheter as compared to an indwelling ureteral stent in the setting of obstruction. 
The authors should be applauded on their efforts in conducting this evaluation, however several limitations are apparent. There is a statistically significant difference in ages between the two groups, as the mean age of the NUS group was 11 years younger than the PIG group. Pain thresholds between individuals of different ages can vary considerably and may impact reporting of pain scores. The authors state that both catheters demanded no great effort in their insertion, although the PIG group was subjectively rated better in terms of ease of catheter placement. The authors did not cite a reason for this observation. If this is the case, then one might conclude that more difficult catheter insertion in the NUS group may translate into greater postoperative discomfort.

While there was no significant difference in selection of calyceal puncture between the groups, $62.5 \%$ of catheters in the PIG group were supracostal,

\section{EDITORIAL COMMENT}

In this prospective randomized trial, the authors evaluated the impact of percutaneous tube configuration $(8.3 \mathrm{~F}$ pigtail nephrostomy tube vs. $8.2 \mathrm{~F}$ nephroureteral stent) on pain scores at postoperative day- 1 and the ease of intraoperative tube placement following percutaneous nephrolithotomy (PCNL) in a total of 18 patients. There were nine patients in each arm. The pigtail group was associated with easier tube placement and better visualization on fluoroscopy (subjective evaluation by the surgeon). Furthermore, patients who received pigtail nephrostomy tubes had marginally less analgesia requirements (morphine equivalent) and their pain scores were significantly lower. There was no statistical difference between compared to only $27.7 \%$ in the NUS group. It is surprising to note that despite a higher number of supracostal catheter placements in the PIG group, the pain scores were lower than in the NUS group. This would suggest that the location of the access does not correlate with the amount of discomfort. The authors appropriately acknowledge that they did not localize the location of pain in either group. This point is extremely important, since pain in the NUS group may be compounded by ureteral and bladder irritation. Without this information, it may be conceivable that even the suture securing each catheter to the skin, may somewhat contribute to the degree of discomfort.

In summary, nephrostomy tube configuration does appear to directly impact postoperative pain. Based on the results provided by the authors, additional studies with larger matched cohorts would serve to validate the conclusions of this study.

Dr. Ravi Munver

Chief, Minimally Invasive Urologic Surgery Director, Endourology Fellowship Program Hackensack University Medical Center Hackensack, New Jersey, USA E-mail:rmunver@humed.com

the two groups regarding the change in hemoglobin and creatinine levels, change in pulmonary function, time to ambulation, and hospital stay. However, patients in the pigtail group resumed oral intake earlier. On the basis of their findings, the authors concluded that the use of an $8.3 \mathrm{~F}$ pigtail nephrostomy tube following PCNL resulted in greater ease of placement and less postoperative pain than an $8.2 \mathrm{~F}$ nephroureteral catheter.

The efficacy and safety of PCNL for the treatment of kidney stones greater than $2 \mathrm{~cm}$ in diameter in upper and middle calices, and greater than $1 \mathrm{~cm}$ in diameter in lower calices, is well established. The ideal drainage method following PCNL is of 
interest, because it is intimately related to the patients' quality of life and length of hospital stay. Currently, there is a trend towards the use of the "tubeless" technique (involving the placement of a ureteral stent instead of a nephrostomy tube) or the use of small bore nephrostomy tubes. Several points relating to the complexity of the procedure, status of the affected kidney, and body habitus of the patient need to be considered before choosing the ideal tube following PCNL. The "tubeless" technique may be appropriate in select patients, such as those with small stone burdens, no significant intraoperative complications and no significant residual stones (1). As such, presence of an intraoperative complication such as significant hemorrhage or perforation, or residual stones mandates placement of a nephrostomy tube. The "tubeless" technique raises concerns that the risk of bleeding and urinary extravasation may be increased. Moreover, the concurrent morbidity of stents should not be underestimated. Additionally, patients with severe hydronephrosis are likely to benefit from the nephrostomy tubes. Lastly, obese patients may suffer from dislodging of the pigtail tubes resulting from pannus movement.

However, "tubeless" PCNL appears to reduce postoperative morbidity. Therefore, it would be useful to examine adjunctive methods that would augment the efficacy and safety of this technique. For example, gelatin matrix thrombin has been used to seal PCNL tracts in small patient series with favorable preliminary results. Lee at al. (2) first described the use of gelatin matrix thrombin in PCNL tracts. However, to date, a prospective randomized trial does not exist in the published literature assessing the true role of hemostatic agents and/or urinary sealants for PCNL tract closure.

As the authors stated, nephroureteral stents may promote more efficient urinary drainage compared to pigtail tubes. Traditionally, drainage after PCNL has been achieved with large bore re-entry tubes to provide effective pelvi-ureteric junction stenting, tamponade of the PCNL tract, preservation of ureteric access, and allow repeat access for a "second look" PCNL if required. However, the current data supports the use of small bore tubes following uncomplicated PCNL (references 4 - 6 in the article). Desai et al. proposed an algorithm for nephrostomy drainage after PCNL based on their large experience and findings of their prospective randomized trial comparing the three different drainage methods (reference 6 in the article). In brief, the authors recommend the use of a large bore (20F) nephrostomy tube following complicated PCNL, and either a 6F double-J stent (if stone-free) or a small bore (9F) nephrostomy tube (if residual stones) following uncomplicated PCNL. Another drainage strategy was proposed by Kim et al. after PCNL for large or complex stones (3). In the presence of pyonephrosis, large residual stones and/or difficult renal anatomy, a $20 \mathrm{~F}$ re-entry tube was recommended. If multiple accesses were required then a $20 \mathrm{~F}$ circle loop was suggested and an $8.5 / 10 \mathrm{~F}$ cope loop was recommended after a standard PCNL.

The authors should be commended for conducting a prospective randomized trial. However, some pertinent issues deserve attention. Ideally, it would be natural to expect a detailed inclusion and exclusion criteria from a prospective study protocol. Secondly, the impact of nephrostomy tube size on stonefree rates with small residual stones after PCNL is not well addressed. From this perspective, it would be useful to know the stone-free rates after PCNL in the current study as well as in future studies on this topic. More importantly, evaluation of the impact of stent/ tube diameter or configuration on the patients' quality of life, as assessed by validated questionnaires, would undoubtedly provide more objective and structured analysis. For instance, Joshi et al. developed and validated the ureteral stent symptom questionnaire (USSQ) for symptom and quality of life evaluation to assess the impact of different types of stents (4). Finally, the sample size estimation for this study does not appear to be based on the primary outcome of interest and the method of power calculation has not been thoroughly described. The only prior study of the three referenced by the authors for power calculation, that has assessed pain as a primary endpoint, recruited 30 patients. Therefore, it is likely that this current study in which 18 patients were recruited was under-powered. Fortunately, the authors found some significant results. The authors reported, for most variables, the means and standard deviations, and used t-tests for data analysis. Medians and inter- 
quartile ranges (IQR) are more appropriate to avoid small means with meaningless negative standard deviations, e.g., IV morphine equivalent with mean = 1 and $\mathrm{SD}=4$. For a study with only 9 patients in each of the two arms, it is important to check assumptions by using t-tests primarily assessing the normality assumption for the continuous variables. Alternatively, nonparametric methods, such as a Wilcoxon test, can be used. Even though this is a randomized study, patient age was different between the two arms. That alone may render the significant difference in the primary outcome artificial.

Notwithstanding these caveats, the current study adds information to the literature with some objective evidence to support the role of small bore pigtail nephrostomy tubes after percutaneous nephrolithotomy in order to reduce operative morbidity.

\section{REFERENCES}

1. Bellman GC, Davidoff R, Candela J, Gerspach J, Kurtz S, Stout L: Tubeless percutaneous renal surgery. J Urol. 1997; 157: 1578-82.

2. Lee DI, Uribe C, Eichel L, Khonsari S, Basillote J, Park HK, et al.: Sealing percutaneous nephrolithotomy tracts with gelatin matrix hemostatic sealant: initial clinical use. J Urol. 2004; 171: 575-8.

3. Kim SC, Tinmouth WW, Kuo RL, Paterson RF, Lingeman JE: Using and choosing a nephrostomy tube after percutaneous nephrolithotomy for large or complex stone disease: a treatment strategy. J Endourol. 2005; 19:348-52.

4. Joshi HB, Newns N, Stainthorpe A, MacDonagh RP, Keeley FX Jr, Timoney AG: Ureteral stent symptom questionnaire: development and validation of a multidimensional quality of life measure. J Urol. 2003; 169: 1060-4.

Dr. Burak Turna

Department of Urology

Ege University School of Medicine

Izmir, Turkey

E-mail: burakturna@gmail.com

\section{REPLY BY THE AUTHORS}

We appreciate the constructive editorial comments of the expert reviews, and would like to clarify the following points. All patients undergoing single access PCNL were offered informed consent - there were no exclusion or inclusion criteria. The USSQ is validated specifically for ureteral stents; it has not been validated for percutaneous nephrostomy tubes. All studies referenced for the power analysis used postoperative pain as a primary endpoint. The one study that randomized 30 patients (ref. 6 in the article) had 10 patients in each arm (large-bore, smallbore, tubeless). 